\title{
Gold nanostructures in biomedical technology
}

\begin{abstract}
Nanomaterials have been defined as any material possessing at least one dimension in the nanometer scale between 1 and $100 \mathrm{~nm}$. As a result, a new field has emerged during last decades: Nanotechnology. It can be defined as the design, synthesis and application of materials and devices whose size and shape have been engineered at the nanoscale. In addition to the main properties of Nanomaterials, Au nanostructures exhibit a variety of optical, physical and chemical properties (localized surface plasmon resonance, photoluminescence, surface-enhanced Raman scattering, resistance, bioinert nature or photo thermal effect) that make them especially interesting for biomedical applications. Here, we will summarize those with high relevance and impact on the future of the medicine in terms of development of new detection and imaging techniques and innovative therapies against cancer.
\end{abstract}

Keywords: gold, nanomaterials, nanotechnology, nanomedicine
Volume 2 Issue 3 - 2017

\author{
Carlos López-Chaves,' Juan Soto Alvaredo, ${ }^{2}$ \\ María Montes Bayón, ${ }^{2}$ Jörg Bettmer, ${ }^{2}$ Juan \\ Liopis,' Cristina Sánchez González' \\ 'Department of Physiology, Faculty of Pharmacy, University of \\ Granada, Spain \\ ${ }^{2}$ Department of Physical and Analytical Chemistry, University of \\ Oviedo, Spain
}

Correspondence: Carlos López-Chaves, Department of Physiology, Campus Cartuja, University of Granada, I807। Granada, Spain, Tel 0034 66570I I75, Email carloschaves@ugr.es

Received: April 19, 2017| Published: May 09, 2017

\section{Introduction}

Gold is mainly known in the jewellery industry due to its tone, rarity and value. However, there are many others advantages (such as bioinert properties ${ }^{1}$ or photo thermal capabilities) ${ }^{2,3}$ that make gold an important material for biomedical applications. Indeed, new therapeutic methods are now being developed by combining nanotechnology and gold. ${ }^{4}$

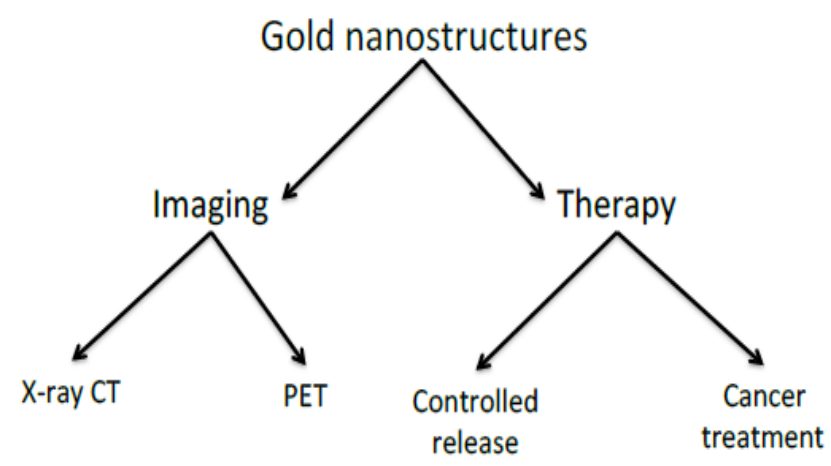

\section{Imaging}

\section{X-ray computed tomography (X-ray CT)}

This technique is one of the most widely used modalities for medical imaging. It provides anatomical information for diagnostics in a cheaper way than other methods. However, the contrast between different types of soft tissues is currently impossible for conventional X-ray CT, so that a good contrast can only be achieved between hard and soft tissues. ${ }^{5,6}$ As a result, conventional X-ray CT cannot be used alone to differentiate cancerous from normal tissues, rendering it essentially useless for the early detection of cancer or cancer metastasis.

As a response to this inconvenient, and given that the attenuation of X-rays by tissue depends on the electron densities, Au provides a strong contrast relative to most of the naturally occurring elements found in the body. ${ }^{7}$ The presence of Au nanostructures can greatly enhance X-ray attenuation, creating high contrast on the CT image. Furthermore, gold nanostructures have shown prolonged presence in the circulation, lower renal toxicity, and well-established surface chemistry for the conjugation of targeting ligands. All these attributes make Au nanostructures promising contrast agents for clinical X-ray CT applications. ${ }^{8,9}$

\section{Positron emission tomography (PET)}

PET is one of the most frequently used modalities in molecular imaging, and can be employed to collect information about the expression of molecular species on a biological tissue. It is based on the detection of pairs of gamma rays emitted indirectly by a positron emitting radionuclide, which is introduced into the body on a biologically active molecule. Thus, by chelating or covalently attaching the radionuclide to the surface of Au nanostructures, doctors are able to visualize those areas where gold nanostructures are accumulated. ${ }^{5,10-12}$

Besides, it is used to obtain valuable information about cellular function and gives insight into the molecular processes of a living organism, enabling diagnosis even before changes at any anatomical are observed.

In addition to this, both techniques may also be combined for multimodality imaging; which gives the opportunity to obtain better results regarding biodistribution, targeted accumulation and clearance of $\mathrm{Au}$ Nanomaterials by joining the morphological and quantitative data acquired from X-ray CT and PET. ${ }^{13-15}$

\section{Therapy}

\section{Drug loading and controlled release}

Being able to load and release a drug in the target tissue (thus considerably reducing side effects), supposes a major step in the development of a medicine. To this purpose, several methods have been published: Recent studies have been focused on the development of so called Au hollow nanostructures. In this way, drugs can be loaded in the interior of these nanostructures to decrease the degradation and increase loading efficacy. Furthermore, by modifying the surface, authors have been able to deliver the drug under controlled circumstances ( $\mathrm{pH}$, temperature-sensitive polymer) or tissues (by attaching specific antibodies to the surface). ${ }^{16}$ 
Additionally, drugs can also be loaded onto the surface of the gold Nanomaterials. For instance, the high affinity between $\mathrm{Au}$ and other atoms like sulphur or nitrogen, the electrostatic interactions or van der Waals forces as well as hydrogen bonding, have showed promising results as tools for drug delivery. ${ }^{17,18}$ In any case, gold nanostructures have shown promising properties in realizing drugs, based on their ability to heat and break chemical bonds between the drug molecule and the Au surface under the irradiation of light..$^{19,20}$ Thus, by combining these techniques, researchers have been able to develop a smart gold nanostructures able to load the drug, travel to the target tissue and deliver the drug.

\section{Cancer therapy}

Based on the ability of gold nanoparticles to heat under light irradiation, photo thermal therapy has gained importance last year's. ${ }^{2,21}$ This process relies on the use of hyperthermia to kill tumour cells (after exposing them to high temperatures for several minutes). However, the nonspecific heating which also destroys adjacent tissues implies a counter indication in this therapy. The fact that these hyper thermal agents are developed in the nanoscale significantly reduces the side effects. Furthermore, it is possible to accumulate gold Nanomaterials at the tumour zone thanks to active targeting enabled by ligands such as antibodies. ${ }^{22,23}$ Thus, the accumulation of Au nanostructures at the desired site will lead to selective heating of target cells after laser irradiation, minimizing potential damage to the surrounding tissue. This advantage, together with the fact that $\mathrm{Au}$ nanostructures can serve as contrast agents for various imaging techniques, would allow physicians to achieve an imaging-guided therapy.

\section{Conclusion}

Being able to quickly detect and treat a disease like cancer with minimal counter indications is a goal that scientists have been pursuing for several years. The huge potential benefit of Au Nanomaterials in the development of nanotechnology and nanomedicine, has contributed to the increased use of these structures as feasible tools in new imaging techniques, drug release and cancer treatment.

\section{Acknowledgements}

The authors gratefully acknowledge the financial support from the Spanish MICINN (Spanish Ministry for Science and Innovation, Grant Number CTQ2011-23038) and MECD (Spanish Ministry for Education, Culture and Sports, Grant Number FPU13/00062). The authors thank JH Thompson for translating the manuscript into English.

\section{Conflict of interest}

Author declares that there is no conflict of interest.

\section{References}

1. Nelson BC, Petersen EJ, Marquis BJ, et al. NIST gold nanoparticle reference materials do not induce oxidative DNA damage. Nanotoxicology. 2013;7(1):21-29.

2. Silva CO, Rijo P, Molpeceres J, et al. Bioproduction of gold nanoparticles for photothermal therapy. Ther Deliv. 2016;7(5):287-304.

3. Rau LR, Huang WY, Liaw JW, et al. Photothermal effects of laser-activated surface plasmonic gold nanoparticles on the apoptosis and osteogenesis of osteoblast-like cells. Int J Nanomedicine. 2016;11:3461-3473.

4. Yang X, Yang M, Pang B, et al. Gold Nanomaterials at Work in Biomedicine. Chem Rev. 2015;115(19):10410-10488.
5. You S, Jung HY, Lee C, et al. High-performance dendritic contrast agents for X-ray computed tomography imaging using potent tetraiodobenzene derivatives. J Control Release. 2016;226:258-267.

6. Lusic H, Grinstaff MW. X-ray-computed tomography contrast agents. Chem Rev. 2013;113(3):1641-1666.

7. Okuno M, Hamaguchi H. Multifocus confocal Raman microspectroscopy for fast multimode vibrational imaging of living cells. Opt Lett. 2010;35(24):4096-4098.

8. Dorsey JF, Sun L, Joh DY, et al. Gold nanoparticles in radiation research: potential applications for imaging and radio sensitization. Transl Cancer Res. 2013;2(4):280-291.

9. Hahn MA, Singh AK, Sharma P, et al. Nanoparticles as contrast agents for in-vivo bioimaging: current status and future perspectives. Anal Bioanal Chem. 2011;399(1):3-27.

10. Xie H, Wang ZJ, Bao A, et al. In vivo PET imaging and biodistribution of radio labeled gold nanoshells in rats with tumor xenografts. Int $J$ Pharm. 2010;395(1-2):324-330.

11. Xie H, Diagaradjane P, Deorukhkar AA, et al. Integrin $\alpha v \beta 3$-targeted gold nanoshells augment tumor vasculature-specific imaging and therapy. Int J Nanomedicine. 2011;6:259-269.

12. Lu W, Melancon MP, Xiong C, et al. Effects of photoacoustic imaging and photothermal ablation therapy mediated by targeted hollow gold nanospheres in an orthotopic mouse xenograft model of glioma. Cancer Res. 2011;71(19):6116-6121.

13. Moses WW. Fundamental Limits of Spatial Resolution in PET. Nucl Instrum Methods Phys Res A. 2011;1:S236-S240.

14. Debbage P, Jaschke W. Molecular imaging with nanoparticles: giant roles for dwarf actors. Histochem Cell Biol. 2008;130(5):845-875.

15. Cole LE, Ross RD, Tilley JM, et al. Gold nanoparticles as contrast agents in x-ray imaging and computed tomography. Nanomed. 2015;10(2):321-341.

16. Yavuz MS, Cheng Y, Chen J, et al. Gold nanocages covered by smart polymers for controlled release with near-infrared light. Nat Mater. 2009;8(12):935-939.

17. Kim D, Jeong YY, Jon S. A drug-loaded aptamer-gold nanoparticle bioconjugate for combined CT imaging and therapy of prostate cancer. ACS Nano. 2010;4(7):3689-3696.

18. Chakravarthy KV, Bonoiu AC, Davis WG, et al. Gold nanorod delivery of an ssRNA immune activator inhibits pandemic H1N1 influenza viral replication. Proc Natl Acad Sci USA. 2010;107(22):10172-10177.

19. Thibaudau F. Ultrafast Photothermal Release of DNA from Gold Nanoparticles. J Phys Chem Lett. 2012;3(7):902-907.

20. Poon L, Zandberg W, Hsiao D, et al. Photothermal release of singlestranded DNA from the surface of gold nanoparticles through controlled denaturating and Au-S bond breaking. ACS Nano. 2010;4(11):6395-6403.

21. Kang S, Bhang SH, Hwang S, et al. Mesenchymal Stem Cells Aggregate and Deliver Gold Nanoparticles to Tumors for Photothermal Therapy. ACS Nano. 2015;9(10):9678-9690.

22. Lee UY, Youn YS, Park J, et al. Y-shaped ligand-driven gold nanoparticles for highly efficient tumoral uptake and photothermal ablation. ACS Nano. 2014;8(12):12858-12865.

23. Li X, Zhou H, Yang L, et al. Enhancement of cell recognition in vitro by dual-ligand cancer targeting gold nanoparticles. Biomaterials. 2011;32(10):2540-2545. 